\title{
Behavior of Sheet Metal Under Multiaxial Deformation Paths
}

\author{
KESTER D. CLARKE ${ }^{1,1,5}$ YURI HOVANSKI, ${ }^{2,6}$ DANIEL R. COUGHLIN, ${ }^{3,7}$ \\ and JOHN E. CARSLEY ${ }^{4,8}$ \\ 1.-Colorado School of Mines, Golden, CO, USA. 2.-Brigham Young University, Provo, UT, USA. \\ 3.-Los Alamos National Laboratory, Los Alamos, NM, USA. 4.-General Motors Company, \\ Warren, MI, USA. 5.—e-mail: kclarke@mines.edu. 6.-e-mail: yuri.hovanski@byu.edu. 7.-e-mail: \\ coughlin@lanl.gov. 8.—e-mail: john.carsley@gm.com
}

The fabrication of complex shapes from sheet metals requires understanding of material behavior across a wide variety of strain states, paths, and rates, and yet material properties are often characterized by a uniaxial tensile test and modeling efforts suffer from limited experimental results to span the desired processing space. Thus, the advanced constitutive models of today require material parameters obtained under multiaxial and complex loading conditions. Over the past few decades, the sheet metal forming community has observed that such advanced constitutive models improve the predictive accuracy on formability and springback. Here, we have assembled articles that address some of the critical areas of need in the understanding of material behavior under complex strain path deformation, including the examination of testing methodologies such as cruciform, tube bulging, elevated temperature hemispherical punch drawing, and cylindrical cup drawing, along with furthering the understanding of local material response in the presence of voids, and the effects of tension leveling parameters on the microstructure in a strip rolling process.

In the first article, "Insights into Cruciform Sample Design," the authors show the importance of specimen design on the level of biaxial strain achievable before failure. The cruciform sample can be used to test materials along nonlinear strain paths, and advanced test systems have been developed that can apply multiaxial, nonmonotonic strain paths and measure stresses and surface strains to determine more effectively the evolution of the yield surface for a given material. Nevertheless, the cruciform sample geometry is not standardized and this article shows that the sample

Kester Clarke is the JOM advisor for the Shaping \& Forming Committee of the TMS Materials Processing \& Manufacturing Division, and guest editor for the topic Behavior of Sheet Metal Under Multiaxial Deformation Paths in this issue. thickness, fillet radius into the reduced section "pocket," and distance from the center of the sample to the notch all have a significant effect on the strain paths and magnitudes obtainable for a given material condition.

Next, the results of a nonambient, hemispherical stretch forming test are reported in "Formability of Magnesium Alloy AZ31B from Room Temperature to $150^{\circ} \mathrm{C}$ When Deformed Under a Biaxial Load," and the authors confirm recent predictions from the literature suggesting the primary deformation mechanism can be changed from twinning to slip in biaxial deformation by increasing temperatures from ambient, even at relatively small strains. The ability to correlate microstructure development with multiaxial deformation strains is critical to enable the creation of informed microstructureaware models that can predict microstructure evolution during complex forming operations, and the understanding of microstructural development during deformation is essential to be able to create new alloys with improved fabrication and performance characteristics.

The article "Effect of Evolutionary Anisotropy on Earing Prediction in Cylindrical Cup Drawing" presents efforts to predict earing continuously during cup-drawing through the measurement of mechanical properties using balanced biaxial hydraulic bulge testing and uniaxial tensile testing of a stainless steel sheet. The authors examine the effectiveness of two yield criteria in predicting anisotropy and modify these criteria by continuously defining yield function coefficients as a function of equivalent plastic strain and plastic work, thereby establishing material condition evolution during forming. These constitutive models were implemented into a user material subroutine of a cup-drawing finite element model, and it was found that the evolutionary approach improved predictions of both earing and local stress distributions in the formed cup. 
In "Modeling of Sheet Metal Forming Based on Implicit Embedding of the Elasto-Plastic Self-Consistent Formulation in Shell Elements: Application to cup drawing of AA6022-T4," the authors show that specific details of microstructure evolution, including texture, anisotropic hardening, the Bauschinger effect, and nonlinear unloading, can be incorporated into finite element analyses of cup drawing through crystal plasticity material models. The simulation results were compared with experimental cup-drawing experiments both in the clamped condition and after springback, and they showed good agreement with respect to the cup geometry, nonuniform residual stress-strain distribution, and crystallographic texture.

The authors of "Characterization of Multiaxial Stress-Strain Response of Tube Metal from DoubleSided Hydro-Bulging Test Based on Hosford's 1979 Yield Criterion" show a somewhat different approach to measuring the mechanical properties of thinwalled tubes under triaxial stress states, and they demonstrated application to the measurement of aluminum alloy 5052-O properties. The results indicate that the resulting uniaxial stress-strain predictions based on various yield criteria vary somewhat from uniaxial tension, but they can be modified by application of reasonable anisotropy coefficients. The use of double-sided hydraulic bulging, i.e., where pressure is applied both internally and externally, shows that altering the loaded stress state can be used to alter the formability of metal sheets.

The application of strain path analyses to a practical, but difficult, manufacturing process is described in "Influence of Tension Leveling Parameters on the Microstructure and Mechanical Properties of Steel Strip." Here, the authors discuss how the variation of tension leveling parameters can affect the microstructure and, as a result, the mechanical properties of steel strips. Optimal balance between strip tension and bending roller offset was determined based on the final measured properties and grain size of the 2.3-mm-thick strip. Since tension leveling plays an important role in improving product quality, and consists of extremely complex through-thickness elastic-plastic deformation, understanding of the subtle changes that result in significant differences in the final product is necessary.

Finally, the extremely complex problem of localized deformation conditions in the presence of voids is considered in "Dilatational Response of Voided Polycrystals." In addition to microstructural features such as grain size, crystallographic texture, or phase fractions, the presence of defects, in this case voids, in a material is particularly difficult to model effectively. The authors present a numerical procedure for performing crystal plasticity finite element analyses of voided, isotropic polycrystals in both tensile and compressive conditions. Although the results are similar to those obtained by von Mises yield surface calculations, specific conditions, namely compressive and triaxial stress states, showed more pronounced differences. In applications where localized strains may initiate failure, these subtle differences may result in significant differences in predicted performance of a material.

The articles assembled here are largely solicited from the TMS2016 symposium "Material Behavior Characterization via Multi-Directional Deformation of Sheet Metal," sponsored by the TMS Shaping \& Forming Committee, and organized by John Carsley, Daniel Coughlin, Myoung-Gyu Lee, Youngung Jeong, and Piyush Upadhyay.

The following articles are published under the topic "Behavior of Sheet Metal Under Multiaxial Deformation Paths" in the May 2017 issue (vol. 69, no. 5) of JOM and can be accessed via the JOM page at http://link.springer.com/journal/11837/69/5/page/ 1link.springer.com:

- "Insights into Cruciform Sample Design," A. Creuziger, M.A. Iadicola, T. Foecke, E. Rust, and D. Banerjee

- "Microstructure Correlation with Formability for Biaxial Stretching of Magnesium Alloy AZ31B at Mildly Elevated Temperatures," Isaac Chelladurai, Michael P. Miles, David T. Fullwood, John E. Carsley, Raj K. Mishra, Irene J. Beyerlein, and Marko Knezevic

- "Effect of Evolutionary Anisotropy on Earing Prediction in Cylindrical Cup Drawing," H.J. Choi, K.J. Lee, Y. Choi, G. Bae, D.-C. Ahn, and M.-G. Lee

- "Modeling of Sheet Metal Forming Based on Implicit Embedding of the Elasto-Plastic SelfConsistent Formulation in Shell Elements: Application to Cup Drawing of AA6022-T4," Milovan Zecevic and Marko Knezevic

- "Characterization of Multiaxial Stress-Strain Response of Tube Metal from Double-Sided Hydro-Bulging Test Based on Hosford's 1979 Yield Criterion," Xiao-Lei Cui, Zhai-Ping Yang, and Xiao-Song Wang

- "Influence of Tension Leveling Parameters on the Microstructure and Mechanical Properties of Steel Strip,” J. Zhang, C.L. Zhou, H.B. Li, X.C. Zhang, and M. Li

- "Dilational Response of Voided Polycrystals," Daniel J. Savage, Oana Cazacu, and Marko Knezevic 